\title{
Dosimetry in Targeted Radionuclide Therapy: The Bad Berka Dose Protocol-Practical Experience
}

\author{
Christiane Schuchardt, Harshad Kulkarni, Carolin Zachert, Richard P Baum
}

\begin{abstract}
Aim: Calculating the absorbed dose is important for the determination of risk and therapeutic benefit of internal radiation therapy. Optimal dose estimations require time-consuming and sophisticated methods, which are difficult due to practical reasons. To make dosimetry available for each of the patients, we developed a specific dosimetry procedure used in daily clinical routine.
\end{abstract}

Materials and methods: Dosimetry has been performed according to the MIRD scheme and adapted to the special conditions at our department (which we have called as the Bad Berka Dose protocol, BBDP): Conjugated planar whole-body scintigraphies at $0.5,3,24,48$ and 72 hours postinjection are analyzed by regions of interest with 'HER MES WHOLE-BODY DISPLAY ${ }^{T M}$ and the time-dependent organ and tumor activities are determined with Microsoft EXCEL ${ }^{\mathrm{TM}}$. The cumulated activity is calculated using the software ORIGIN PRO $8.1 \mathrm{G}^{\mathrm{TM}}$ and a mono- or biexponential fit of the time-activity curves. Mean absorbed doses are finally estimated using the software OLINDA EXM ${ }^{\mathrm{TM}}$.

Results: We found a compromise between the calculation model and practical conditions. It has ensured dose estimation in daily clinical routine with a reasonable effort and within acceptable time. In consequence, the dosimetry method developed for Bad Berka allows each of our patients to undergo dosimetry after therapy using Lu-177-labeled peptides (peptide receptor radionuclide therapy). Additionally, this approach can be used for any internal radiotherapy using a gamma-emitting radionuclide.

Conclusion: The BBDP is a practicable dosimetric approach, which can be used in daily clinical routine. It not only helps in identifying patients who would benefit most from the treatment, but also those with unfavorable dosimetry. Additionally, the analysis of dosimetric data from peptide receptor radionuclide therapy (PRRNT) could help in predicting possible toxicity.

Keywords: Dosimetry, Peptide receptor radionuclide therapy, DOTATATE, DOTATOC.

How to cite this article: Schuchardt C, Kulkarni H, Zachert C, Baum RP. Dosimetry in Targeted Radionuclide Therapy: The Bad Berka Dose Protocol-Practical Experience. J Postgrad Med Edu Res 2013;47(1):65-73.

\section{Source of support: Nil}

Conflict of interest: None declared

\section{INTRODUCTION}

Currently, various radiolabeled therapeutic agents are used against different forms of cancer. The challenge of internal targeted radionuclide therapies is to deliver the highest possible dose to the tumor while sparing normal organs from damage. For the determination of the risk and therapeutic benefit of such internal therapies, patient-specific dosimetry is an essential prerequisite.

Dosimetry represents meanwhile a precious guide for the selection of radionuclides and peptides as well as for therapy optimization. There exist various methods to estimate internal doses and recent studies promise new perspectives to come. ${ }^{1}$ Furthermore, a recent publication presents guidelines regarding optimal practice of internal dosimetry which would also enable researchers to use the information for possible improvements to the approach. ${ }^{2}$

Dose estimations could be based on either twodimensional (2D) planar gamma camera imaging or threedimensional (3D) imaging using SPECT/CT or positron emission tomography/computed tomography (PET/CT)., ${ }^{3,4}$ The 3D techniques to enable the absorbed dose calculation are however not routinely available. In fact, the MIRD scheme provides a more conventional method for cal culating absorbed doses of radionuclides. ${ }^{5}$ The optimal dose estimation requires time-consuming and sophisticated methods, including pharmacokinetic biodistribution as well as washout studies using the same radionuclide as is used for therapy. This may be difficult owing to practical (e.g. the patients' status) and physical reasons.

Due to encouraging clinical results, peptide receptor radionuclide therapy (PRRNT) with radiolabeled somatostatin analogs (SSTA) is now established as a treatment modality in gastroenteropancreatic (GEP) neuroendocrine tumors (NETs) and therefore one of the most frequently used targeted radiotherapies. Because of an increasing number of PRRNTs, we aimed to make dosimetry available for most of the patients and developed a specific dosimetry procedure. The Bad B erka D ose protocol (BB DP) is based on planar whole-body scintigraphies and used in our daily clinical routine. The estimated mean absorbed doses to organs and tumor lesions obtained from these dosimetric calculations can be used to evaluate therapy response as well as possible adverse effects.

\section{Bad Berka Dose Protocol}

The dose estimation requires an accurate determination of the time-dependent activity of the source regions. Thus, most important is the correct evaluation of the distribution and 
the kinetics of the administered radiopharmaceutical. ${ }^{6,7} \mathrm{~F}$ or dose estimations we developed a convenient procedure which is based on the MIRD scheme, using the OLINDAEXM ${ }^{T M}$ software by adapting the calculation model to our special conditions. The main objective was to establish a method which is practicable in our daily clinical routine for a huge number of patients. ${ }^{8,9} \mathrm{Flow}$ Chart 1 show the BBDP.

The following camera parameters were used for planar whole-body imaging: MEDISO spirit DH-V dual-headed gamma camera (M edical Imaging Systems, Budapest, Hungary), M eG P collimator, 15\% energy window, peak at $208 \mathrm{keV}$, scan speed $15 \mathrm{~cm} / \mathrm{min}$. Whole-body scintigraphies were acquired at 5 time points postinjection: 0.5 hours (immediately after administration of therapeutic activity) followed by 3, 20, 44 and 68 hours postinjection.

The dose estimation consists of four main steps:

1. ROI analysis: Manual drawing of regions of interest (ROI) using the HERMES TM whole-body display (Hermes M edical Solutions, Stockholm, Sweden).

2. Determination of activity: A nalysis of ROI statistics using Excel sheet.

3. Fit: Fit of the time-activity graph using ORIGIN PRO $8.1 \mathrm{G}^{\mathrm{TM}}$

4. Dose estimation using OLINDAEXM $1.1^{\text {TM }}$.

The organs showing tumor involvement or overlaying with other source regions were excluded from dosimetric evaluation. For this reason, normal liver was not included in the analysis in this study because nearly all patients had extensive liver metastases. Some patients had liver lesions superimposing on the right kidney, allowing only analysis of the left kidney. In these cases, it was assumed that the mean absorbed dose would be identical for both kidneys (which were also checked and confirmed by prior ${ }^{99 \mathrm{~m}} \mathrm{Tc}$ MAG3 scintigraphy proving that there was no significant difference in the differential renal function). A Iso, kinetics and mean absorbed dose to the spleen were not estimated in several patients, who had undergone splenectomy.

To estimate the mean absorbed dose to red marrow (RM), blood sampling was performed. After the administration of the therapeutic dose, venous blood samples were obtained at different time points postinjection. The radioactivity in $0.5 \mathrm{ml}$ blood samples was measured using a high-purity germanium detector and the activity in $\mathrm{M} \mathrm{Bq} / \mathrm{ml}$ was plotted against time. Depending on the degree of correlation, the curves were fitted to bi- or triexponential functions to determine the cumulated activity in blood. Assuming that there was no specific uptake in the blood cells, a uniform distribution in the blood, and that clearance from RM was equal to that from the blood, the mean absorbed dose to RM was estimated by using the S-values from the software OLINDA EXM TM 10,11

All data as well as dosimetric parameters and results were documented. The database al so contains additional data (e.g. concerning pretherapy examinations) which facilitates comprehensive individual and/or interindividual analysis.

A possible drawback of the BBDP may be that it is based only on $2 \mathrm{D}$ planar imaging. It is known, that there are intrinsic limitations of these 2D approaches, especially regarding attenuation and scatter correction as well as background and organ overlay. ${ }^{12}$ Sandstöm et al analyzed the feasibility and reliability of individualized dosimetry based on SPECT/CT in comparison with conventional planar imaging in patients treated with ${ }^{177} \mathrm{Lu}$ D OTA TOC. Their results showed that planar and SPECT doses were comparable in areas free of tumors, but that planar dosimetry overestimated the absorbed dose in tumor lesions. ${ }^{13}$ Furthermore, Garkavij et al compared three different quantification methods to evaluate the absorbed dose to the kidneys. They found that patients evaluated according to the conventional planar-based dosimetry may have been undertreated when compared to the evaluation according to other methods using SPECT/CT, because the mean absorbed dose to the kidneys was overestimated. ${ }^{14}$ Even though the kidney dose is overestimated with planar imaging, it is definitely better to be on the safer side than to underestimate and overtreat. In conclusion, in spite of the availability of more accurate methods for dose estimation, planar imaging continues to be the most feasible option.

\section{Dosimetry in Peptide Receptor Radionuclide Therapy}

M ost frequently applied targeted radiotherapy at our department is PRRNT using ${ }^{177} \mathrm{Lu}$ - or ${ }^{90} \mathrm{Y}$-labeled SSTA . B ecause the nuclide ${ }^{177} \mathrm{Lu}$, in contrast to ${ }^{90} \mathrm{Y}$, is not a pure $\beta$-emitter and has al so a certain amount of gamma emission, it can be directly used for imaging and dosimetry during the therapy cycle. Therefore, the BBDP is mainly used for patients receiving ${ }^{177} \mathrm{Lu}$-labeled peptides for therapy.

Since, different subtype receptor affinity profiles of the various SSTA result in different uptake and kinetics in normal tissues and tumors, we compared dosimetric parameters in PRRNT using DOTA TA TE, DOTA TOC and DOTANOC.

To describe differences between the various radiolabeled peptides, the following parameters were chosen: U ptake at 20 hours postinjection (maximum uptake for tumor lesions), effective half-life and mean absorbed dose. To describe differences among the peptides, nonparametric tests were 


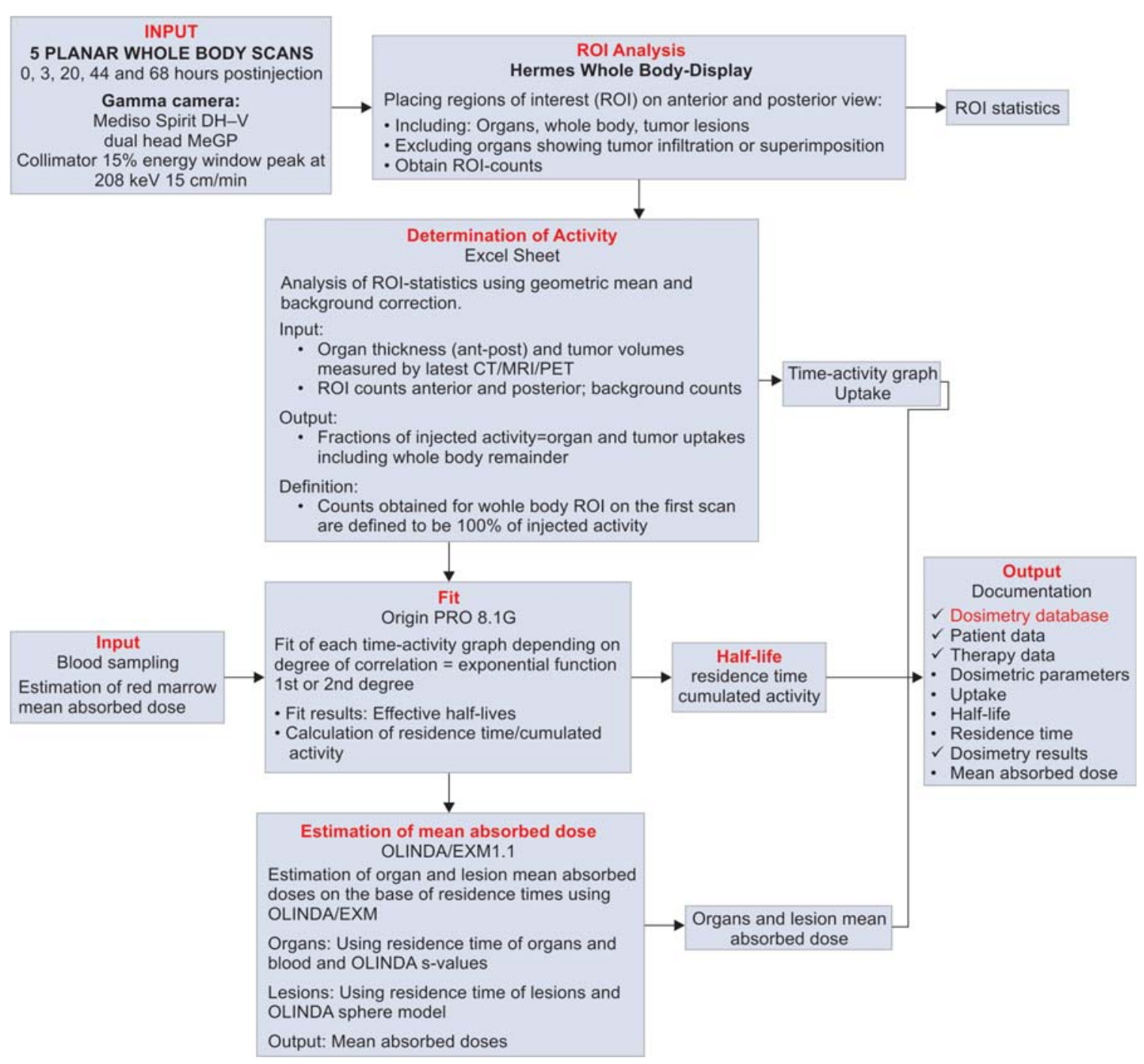

Flow Chart 1: Bad Berka dose protocol

used. All statistical tests were performed on ORIGINPRO $8.1 \mathrm{G}^{\mathrm{TM}} ; \mathrm{p}$-values $\leq 0.05$ were considered to be significant.

A ll patients enrolled in studies concerning PRRNT were suffering from metastatic NETs with liver, lymph node, bone or other organ involvement. Intense somatostatin receptor expression of (inoperable) primary tumors and metastases had been verified before therapy by using ${ }^{68} \mathrm{Ga}$ DOTA NOC, DOTATOC or DOTATATE PET/CT. B eforePRRNT, each patient was extensively informed about the therapeutic procedure and possible adverse effects. All patients provided written informed consent to undergo treatment and follow-up. The studies were approved by the local Ethics Committee and performed in accordance with German regulations concerning radiation safety.

\section{Dosimetry in Peptide Receptor Radionuclide Therapy using ${ }^{177} \mathrm{Lu}$-DOTATATE, DOTATOC and DOTANOC}

The dosimetric parameters from 253 patients, treated with 1 to 6 cycles of ${ }^{177}$ Lu-labeled DOTA TATE, DOTANOC or
DOTATOC were compared. Differences with respect to kinetics, biodistribution and mean absorbed dose, between the three different peptides were analyzed on the basis of dosimetric data obtained in this group.

For normal organs (whole-body, kidneys, spleen), DOTATOC shows the lowest and DOTA NOC, the highest uptake. The mean absorbed organ doses and half-lives were observed to be the lowest for DOTA TOC. In contrast, the highest uptake in tumor lesions was found for D OTA TA TE and the lowest, for DOTANOC. The resulting mean absorbed lesion doses were the highest for DOTATATE, followed by DOTATOC and DOTANOC. DOTATOC was found to have the best tumor to kidney ratio compared to DOTATATE and DOTANOC, apart from the lowest absolute mean absorbed renal dose.

\section{Dosimetry in Peptide Receptor Radionuclide Therapy using ${ }^{177}$ Lu-DOTATATE and DOTATOC in the Same Patient}

For intraindividual comparison, 25 patients who received PRRNT, first using ${ }^{177} \mathrm{Lu}$-D OTA TATE, and in a following 



Graph 1: Comparison of ${ }^{177}$ Lu DOTATATE and ${ }^{177}$ Lu DOTATOC for uptake at 20 hours postinjection and mean absorbed dose in whole body, kidneys and tumor lesions in the same patient

cycle using ${ }^{177} \mathrm{Lu}$-DOTATOC, were included in a study to compare kinetics and mean absorbed doses of the two peptides. The mean time between these therapy courses was 18 months. In case a patient underwent more than one cycle with each peptide, two consecutive cycles were chosen for dosimetric analyses. Graph 1 shows comparative dosimetric results for uptake at 20 hours postinjection and mean absorbed dose.
A higher whole-body uptake at 20 hours postinjection was found for DOTA TATE as compared to DOTA TOC in 24 out of the 25 patients ( $96 \%$ ). The first effective half-life was longer for DOTATOC in 22 patients $(88 \%)$, whereas for DOTATATE the second effective half-life was longer in $17 / 25(68 \%)$ patients. In 22 patients (88\%), whole-body dose was slightly but statistically significantly higher when using DOTATA TE as compared to DOTATOC. 
Renal dosimetric parameters (uptake, residence time and mean absorbed renal dose) were significantly higher for DOTATA TE in 19 of 22 patients ( $86 \%$ ). The effective halflife was found to be similar for both peptides.

A higher uptake of DOTATATE at 20 hours postinjection was observed in $85 \%$ of the analyzed tumor lesions and $50 \%$ had a longer effective half-life of DOTATATE. The mean absorbed dose to the lesions was higher for DOTATATE in $65 \%$ of the lesions. These differences were statistically significant for uptake and mean absorbed dose, but not significant concerning the effective half-life.
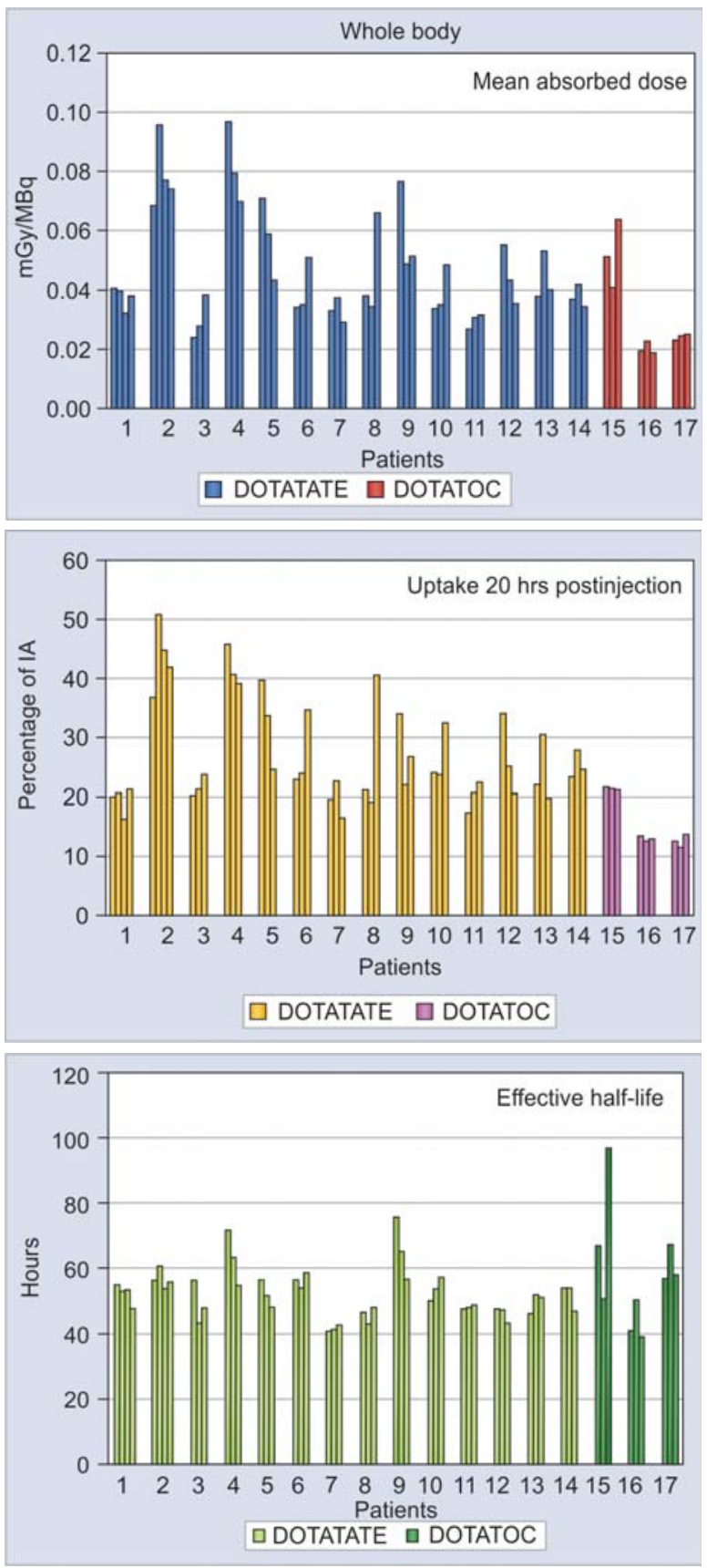

\section{Serial Dosimetry}

Graph 2 shows dosimetric results in 17 patients, which received three or four cycles of ${ }^{177} \mathrm{Lu}$-DOTATA TE or ${ }^{177} \mathrm{Lu}$ DOTATOC. The administered activity was 3 to $8 \mathrm{GBq}$ ${ }^{177} \mathrm{Lu}$ - DOTATA TE or DOTA TOC per cycle. 12/17 patients received 3 cycles of ${ }^{177} \mathrm{~L}$ u-DOTATA TE, $3 / 17$ patients were treated using three cycles of ${ }^{177}$ Lu-DOTATOC and 2/17 patients underwent therapy using four cycles of ${ }^{177} \mathrm{LU}$ DOTATATE.

The mean absorbed renal doses showed a wide range. A low variation was found for the effective half-life, whereas the uptake and mean absorbed dose showed higher
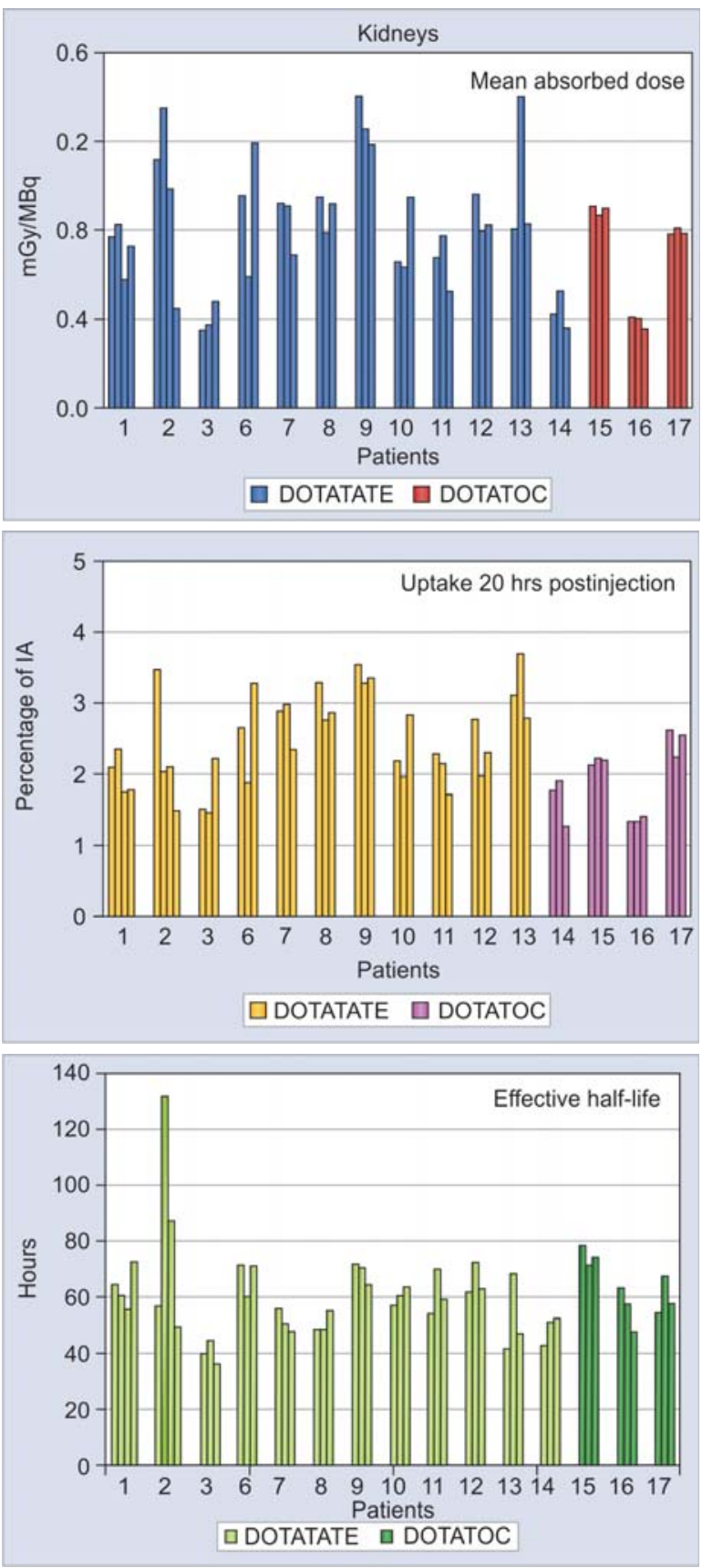

Graph 2: Serial dosimetry results for whole body and kidneys in 17 patients receiving 3 to 4 cycles of ${ }^{177}$ Lu-DOTATATE and ${ }^{177}$ LuDOTATOC. The mean absorbed dose, uptake at 20 hours postinjection and effective half-life are given in mGy/MBq, IA(\%) and hours respectively 


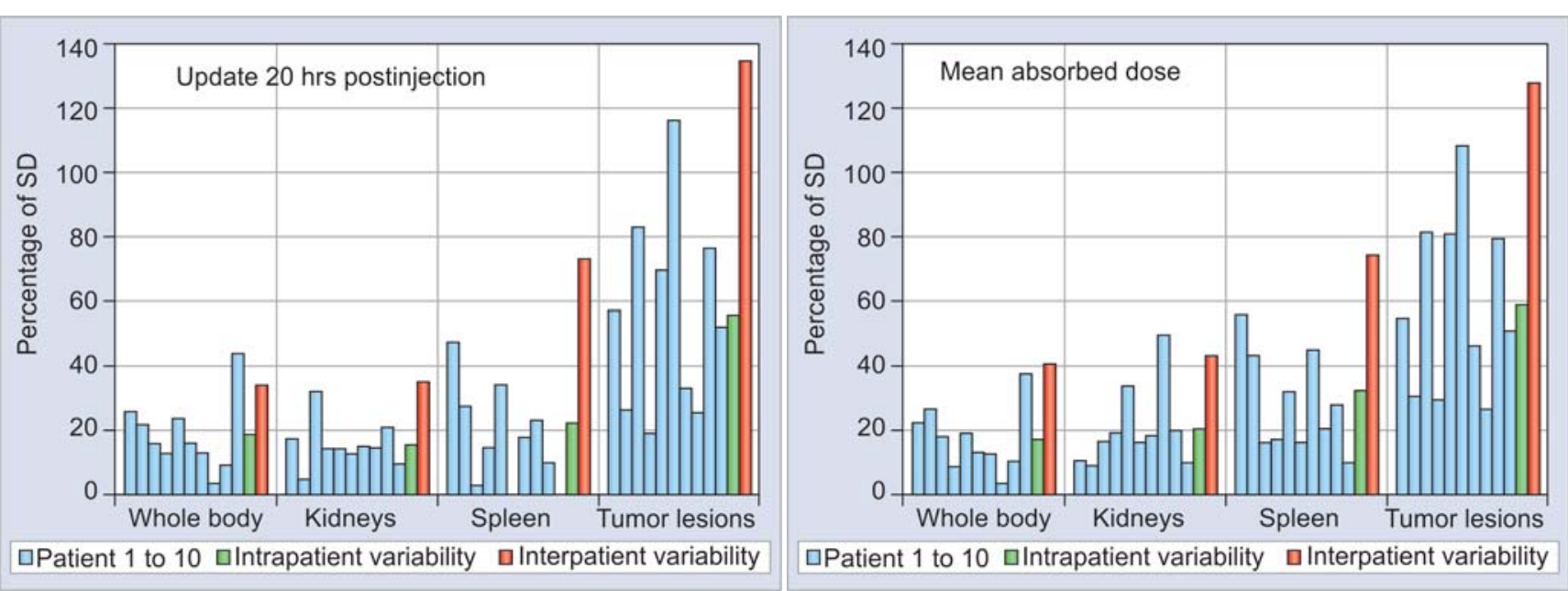

Graph 3: Intraindividual variability (10 patients) and interindividual variability (173 patients) for uptake at 20 hours postinjection and mean absorbed dose represented as percentage of SD (standard deviation)

differences between therapy cycles. Some of the dosimetric results of whole-body showed ascending or descending order in serial cycles, but for most of the dose parameters no systematic pattern was found in consecutive therapy courses.

A lower variation was found for ${ }^{177} \mathrm{Lu}$ U-DOTATOC in the effective half-life, uptake at 20 hours postinjection and mean absorbed dose to whole-body and kidneys values as compared to ${ }^{177} \mathrm{Lu}$-DOTATATE.

\section{Variability}

A high interpatient variability was found for all dosimetric results. This is not unexpected since it was a heterogeneous group of patients having varying receptor densities and tumor burden. In addition, the results showed a high intrapatient variability in the undergoing several cycles of therapy with different peptides.

Graph 3 shows both intra- and interindividual variability for uptake and mean absorbed dose: The variation is given in percent of the standard deviation (\%SD $=\mathrm{SD} /$ mean value). The intraindividual variation was determined using dosimetric parameters from 10 patients which received three therapy cycles of ${ }^{177}$ Lu-D OTA TATE. Based on dosimetric analysis in 173 patients treated with ${ }^{177} \mathrm{Lu}$-DOTATATE, the interindividual variation was determined. The lowest variation was seen for whole-body and kidneys, whereas tumor lesions showed the highest variability. The interpatient variability was higher than the intrapatient variability.

Graph 4 shows dosimetric results in one patient, who received six cycles of therapy using three different peptides. The highest whole-body uptake was observed during the first therapy when using D OTA N OC, while the highest renal uptake was found at third therapy. For the liver lesions, maximum uptake was observed during the first two therapies. DOTA TOC demonstrated the low est whole-body uptake. Also noticeable were the differences in the initial renal uptake, similar effects being seen for liver lesions and the spleen. There was no systematic pattern of uptake or mean absorbed dose in consecutive therapies. A Iso in other patients who received more than one cycle of therapy using ${ }^{177}$ Lu-DOTATATE, kinetics was variable and no specific order for consecutive therapies was found. Consequently, the dosimetric calculations in one cycle of PRRNT should not be used to predict doses during following cycles, even if the same peptide is used.

Although the variability may be attributed to the difference in biological behavior of the peptides, the fact that there might also have been an influence of previous radiopeptide therapies or other treatment modalities, must be taken into account. The possible effects of the previous treatments on the outcome of PRRNT (e.g. effect on the tumor radiosensitivity) are well known in literature.

\section{Conclusion}

BBDP in peptide receptor radionuclide therapy: From the studies concerning comparison of dosimetric results in PRRNT using ${ }^{177} \mathrm{~L}$ u-labeled peptides DOTATATE, DOTANOC and DOTATOC, the following conclusions could be drawn:

- The in vitro higher affinity of DOTANOC correlates with the in vivo higher uptake for whole-body and normal tissue, which results in a higher whole-body dose. Therefore, this peptide is not ideal for PRRNT.

- Concerning kidney uptake and mean absorbed dose to normal organs and whole-body, DOTATOC revealed the highest tumor-to-kidney ratio and is very appropriate for PRRNT. 


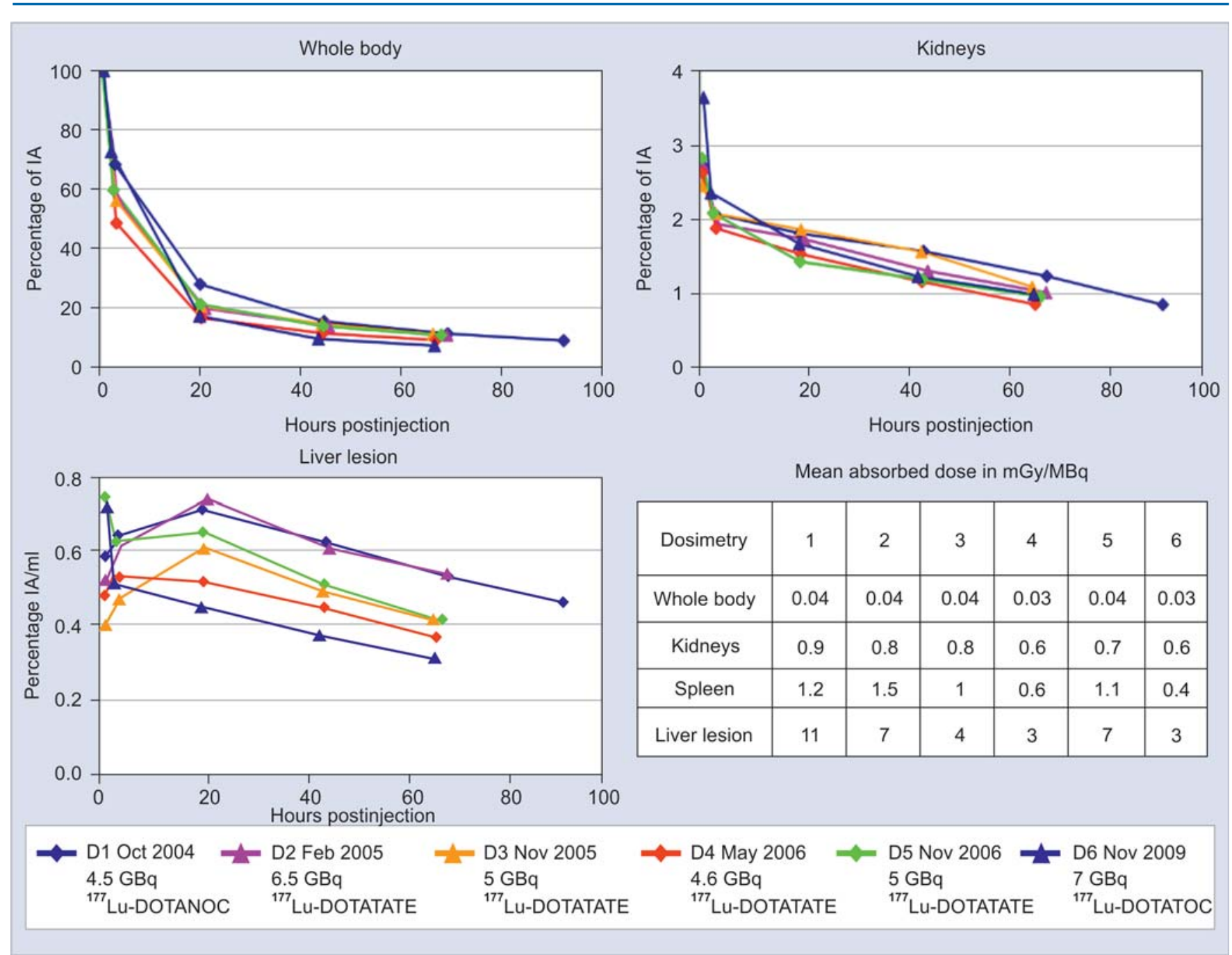

Graph 4: Dosimetric results in 6 cycles of PRRNT in one patient using three different peptides

- DOTATATE was shown to deliver the highest tumor dose (due to the longer residence time in the malignant lesions) and is also very suitable.

A dditionally, the finding of large variability should be addressed in further studies. It is recommended that median values of absorbed doses among patients should not be the only criteria to plan PRRNT. The interindividual differences, particularly organ functionality, metabolism or receptor density in organs and tumor lesions, must be taken into account.

The studies demonstrate further, that the calculation of mean absorbed doses to critical organs and tumor lesions should be considered for estimation of possible toxicity from PRRNT. In conclusion, individual dosimetry in PRRNT is essential for optimal PRRNT.

\section{More Possibilities for the use of the BBDP}

In addition, the BBDP can be used to estimate dosimetric parameters of other radiopharmaceuticals used for internal radiotherapy.
${ }^{177}$ L u-B PA M D ((4- \{[bis-(phosphonomethyl)) carbamoyl ] methyl \}-7, 10-bis (carboxymethyl)-1, 4, 7, 10-tetraazacyclododec-1-yl acetic acid) is a promising new treatment option for skeletal metastases in prostate cancer. W e used the B BDP to determine organ and tumor kinetics and to estimate the mean absorbed dose to normal organs and tumor lesions.

Graph 5 shows the time-activity graphs for the wholebody and the kidneys, and blood kinetics are shown in Graph 6. A fast clearance of ${ }^{177} \mathrm{~L}$ U-BPAM D from wholebody, normal organs as well as from blood was found. In contrary, skeletal lesions showed a very long retention / half-life of the radiopharmaceutical.

W e concluded therefore, that ${ }^{177}$ L u-B PA M D has optimal characteristics for radionuclide therapy of osteoblastic bone metastases in prostate cancer.

\section{CONCLUSION}

W e found a compromise between the calculation model and practical conditions by adapting the MIRD scheme to the 


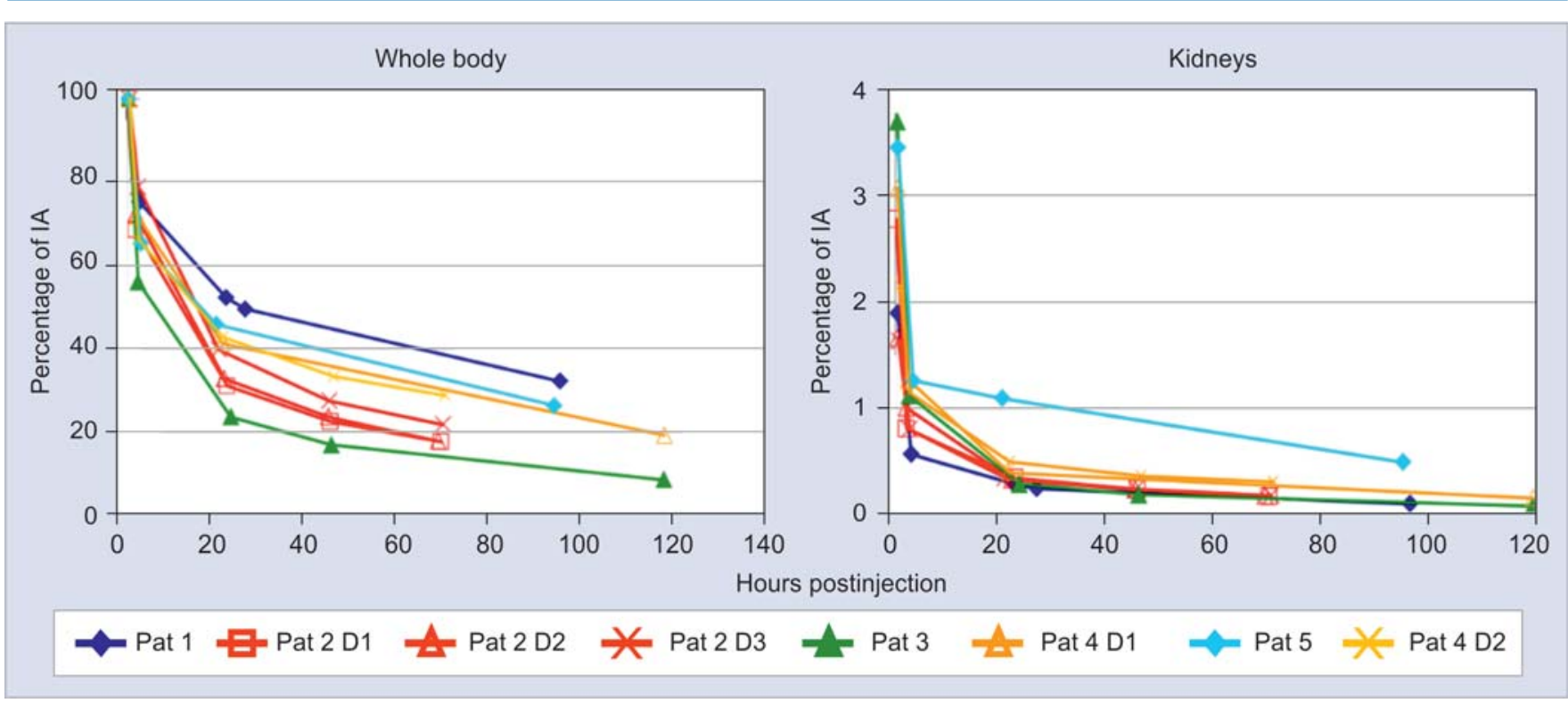

Graph 5: ${ }^{177}$ Lu-BPAMD kinetics (P_patient; D—dosimetry) in five patients receiving 1 to 3 cycles of therapy

special conditions at our department. Although it is known that the quantification of the activity in different organs from planar data is hampered by inaccurate attenuation and scatter correction as well as because of background and organ overlay, the BBDP procedure is very practical approach. We showed that the dosimetric eval uation using the BBDP:

- Helps in identifying SSTA, which are preferable for PRRNT with Lu-labeled peptides.

- Helps to explain possible toxicity, e.g. renal, as the kidneys are dose limiting organs.

- Plays an important role in understanding therapy response and benefit.

The BB DP has ensured dose estimation in daily clinical routine with a reasonable effort and within acceptable time. In consequence, this approach allows each of our patients to undergo dosimetry after therapy using ${ }^{177} \mathrm{Lu}$-labeled peptides.

Since, advanced methods for dose estimations based on 3D imaging using SPECT/CT and appropriate software are not routinely available, the BBDP remains an al ternative solution for internal dose estimation.

Additionally, the BBDP can be used for any internal radiotherapy using a gamma-emitting radionuclide.

\section{REFERENCES}

1. Cremonesi M, Ferrari M, Di Dia A, B otta F, De Cicco C, B odei $L$, et al. R ecent issues on dosimetry and radiobiology for peptide receptor radionuclide therapy. Q J Nucl Med Mol I mag 2011;55(2):155-67.

2. Lassmann $M$, Chiesa $C$, Flux $G, B$ ardiès $M$. EA NM Dosimetry Committee guidance document: Good practice of clinical

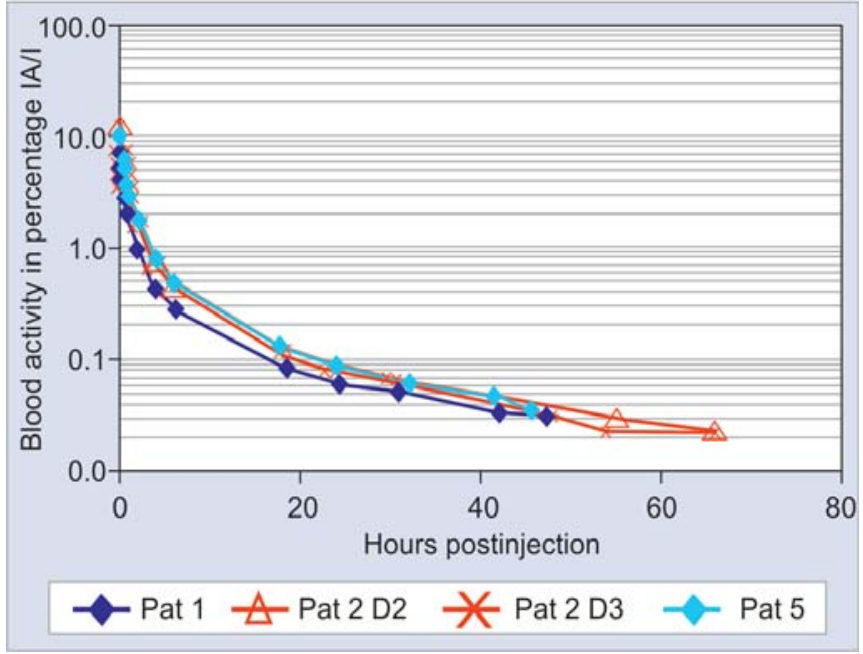

Graph 6: Blood kinetics of ${ }^{177}$ Lu-BPAMD (P-patient; $\mathrm{D}$-dosimetry)

dosimetry reporting. Eur J N ucl M ed M ol Imag 2011;38: 192-200.

3. Sgouros G, Frey E, W ahl R, Bin H, Prideaux A, Hobbs R. 3-D imaging based, radiobiological dosimetry. Semin Nucl Med 2008;5:321-34.

4. Sandström M, Garske U, G ranberg D, Sundin A, L undqvist H. Individualized dosimetry in patients undergoing therapy with ${ }^{177}$ Lu-DOTA-D-Phe1-Tyr3-octreotate. Eur J Nucl Med Mol Imaging 2010;37:212-25.

5. Siegel JA, Thomas SR, Stubbs JB. MIRD Pamphlet 16: Techniques for quantitative radiopharmaceutical biodistribution data acquisition and analysis for use in human radiation dose estimates. J Nucl Med 1999;40:37S-61S.

6. Sgouros G. Dosimetry of internal emitters. J Nucl Med 2005;46:18S-27S.

7. Stabin M G, Siegel JA. Physical models and dose factors for use in internal dose assessment. Health Phys 2003;85(3):294-310. 
8. Wehrmann C, Senftleben S, Zachert C, M ueller D, B aum RP. Results of individual patient dosimetry in peptide receptor radionuclide therapy with ${ }^{177} \mathrm{Lu}$ DOTA-TATE and ${ }^{177} \mathrm{Lu}$ DOTAN OC. Cancer Biother Radiopharm 2007;22(3):406-16.

9. Stabin M G, Sparks RP, Crowe E. OLINDA/EXM : The secondgeneration personal computer software for internal dose assessment in nuclear medicine. J N ucl M ed 2005;46:1023-27.

10. Wessels BW, Bolch WE, B ouchet $L G$, et al. Bone marrow dosimetry using blood-based models for radiolabeled antibody therapy: A multi-institutional comparison. J Nucl Med 2004; 45:1725-33.

11. Sgouros $G$. B one marrow dosimetry for radioimmunotherapy: Theoretical considerations. J Nucl Med 1993;34:669.

12. Bardies M, Buvat I. Dosimetry in nuclear medicine therapy: What are the specifics in image quantification for dosimetry? Q J Nucl Med Mol Imag 2011;55(1):5-20.

13. Sandström M, Garske $U, G$ ranberg $D$, Sundin A , L undqvist $H$. Individualized dosimetry in patients undergoing therapy with ${ }^{177}$ L u-DOTA-D-Phe1-Tyr3-octreotate. Eur J Nucl Med Mol Imag 2010;37:212-25.

14. Garkavij M, Nickel M, Sjögreen-Gleisner K, Ljungberg M, et al. ${ }^{177}$ Lu-[DOTA 0,Tyr3] Octreotate therapy in patients with disseminated neuroendocrine tumors: A nalysis of dosimetry with impact on future therapeutic strategy. Cancer 2010;116(4 Suppl):1084-92.

\section{ABOUT THE AUTHORS}

\section{Christiane Schuchardt}

Medical Physicists, THERANOSTICS Center for Molecular Radiotherapy and M olecular Imaging, ENETS Center of Excellence Zentralklinik Bad Berka, Germany

\section{Harshad Kulkarni}

Resident Physician, THERANOSTICS Center for Molecular Radiotherapy and M olecular Imaging, ENETS Center of Excellence Zentralklinik Bad Berka, Germany

\section{Carolin Zachert}

Senior Physician, THERANOSTICS Center for Molecular Radiotherapy and M olecular Imaging, ENETS Center of Excellence Zentralklinik Bad Berka, Germany

\section{Richard P Baum (Corresponding Author)}

Chairman and Clinical Director, THERAN OSTICS Center for M olecular Radiotherapy and M olecular Imaging, ENETS Center of Excellence Zentralklinik Bad Berka, 99437, Bad Berka, Germany, Phone: +49-364 585-2200, Fax: +49-364-585-3515, email: richard.baum@ zentralklinik.de www.zentralklinik-bad-berka.de 